\title{
The effectiveness of the cervical halo: open versus closed ring. A preliminary report
}

\author{
FT Wetzel ${ }^{1}$, NW Dunsieth, $\mathrm{Jr}^{2}$, KR Kuhlengel ${ }^{3}, \mathrm{EM} \mathrm{Paul}^{2}$ and DM Lahey ${ }^{2}$ \\ ${ }^{1}$ Associate Professor and Director, University of Chicago Spine Center, 4646 North Marine Drive, Chicago, \\ Illinois 60640; Departments of ${ }^{2}$ Orthopaedics and Surgery, ${ }^{3}$ Division of Neurosurgery, The Pennsylvania State \\ University, College of Medicine, The Milton S Hershey Medical Center Hershey, Pennsylvania, USA
}

\begin{abstract}
The halo cervical orthosis has proven extremely effective in stabilizing the spine, both non-operatively and as a supplement to operative procedures. Current designs of the available halo utilize either a closed or an open stabilizing ring. Twenty-four patients with various indications for halo application are reviewed. Eleven were treated with a closed ring apparatus (Ace Medical, Los Angeles, California), and thirteen with an open ring device (Bremer, Inc, Jacksonville, Florida). X-rays of the treated patients were compared by group, and patients were interviewed regarding their complaints while wearing the halo. Rates of complication were compared. Results showed no significant differences between radiographs (kyphosis or translation) throughout the follow-up period. Patients experienced a significantly higher incidence of halo-associated pain in the open group. Otherwise, there were no statistical differences in the complication rates of either device. Whether or not the higher incidence of pain in the open group is related to decreased device rigidity is unknown. The open design may theoretically permit bending and opening of the ring to occur, the so-called 'wishbone' effect. Based on these data, it cannot be determined whether the advantages of the open ring-ease of application-are offset by this potential disadvantage. Clearly, a larger, randomized prospective study is required to investigate this.
\end{abstract}

Keywords: halo cervical orthoses; spinal stabilization

\section{Introduction}

The halo cervical orthosis was first described by Perry and Nickel in 1959. ${ }^{1}$ The original model consisted of a rigid metal ring attached to the skull with threaded pins. The ring was supported by metal uprights with their bases fixed in a plastic mould that was cast around the shoulder and chest of the patient. Most cervical orthoses tried to achieve tension through the use of less stable structures at the lower contour of the head. The halo, however, was purported to pull the neck 'like the spoke of a bicycle wheel' to achieve its desired effect. The technique met with great success, achieving better overall immobilization of the cervical spine than any other cervical orthotic device, especially in rotation. There still remains some debate as to whether or not the individual vertebrae are immobilized with the halo. Koch and Nickel noted that segments moved an average of $31 \%$ of normal in flexion extension during halo immobilization, ${ }^{2}$ Anderson et al noted that $7^{\circ}$ of angulation and $1.7 \mathrm{~mm}$ of translation were permitted at injured levels, with halo immobilization in place. ${ }^{3}$ Some observers have reported alternating flexion and extension between vertebrae with attempted sagittal movement in the device. When summed, however, this 'snake-like' movement results in no net motion and is thought to be insignificant. ${ }^{4}$

The fundamental halo design has remained intact.
One of the components subject to modification, however, has been the ring. There have been a number of attempts to improve design for better performance and practicality. Traditionally, the halo ring has simply been a flattened circle of metal with pin holes and attachments for the upright posts. Bremer (Jacksonville, Florida) introduced the first 'open ring' design in which the halo ring was discontinuous on a single plane. This ring has a curved supporting bar lying in a plane perpendicular to the major component of the ring. Structurally, this allows the rear portion of the flat ring to remain open, thus making the devise easier to apply. An open ring may flex anteriorly when spread; thus, stability has been a concern with the open design. This preliminary study, therefore, was designed to compare the open ring with a more traditional closed ring design, in terms of stability and comfort.

\section{Methods and materials}

\section{Patient selection}

Twenty-four consecutive patients are included. All were treated with a halo for cervical instability or postoperative support. No patient had an injury below T1, and all were followed up for at least 3 months following halo removal, in order to assess stability whilst 'in halo' and immediately following its removal. 
Eleven patients were treated with a closed ring, and 13 were treated with an open ring.

Following halo placement, all patients were instructed in pin site care. Cleaning of pin sites with half strength hydrogen peroxide twice daily was recommended, to keep pin sites clean. Initially, this was followed by application of Betadine ointment. Pin loosening rates using the criteria of Garfin et $a l^{5}$ were determined by chart review or upon examination.

\section{Radiographic evaluation}

Serial radiographs, including pre-application, halo in place, and post-halo removal were reviewed. The radiographs were placed on a light source and overlaid with tracing paper. Landmarks for kyphotic ankle and translation were traced from the neutral views of the cervical spine. These tracings were labelled by patient, date of follow-up, and level of injury. Values for kyphotic ankle and translation were obtained by digitizing the tracing. Accuracy was determined by serial digitization of two groups of films ten times. Translation and kyphosis were determined with translation measurements recorded to one-tenth of a milimeter, and kyphotic angle recorded to one-thousandth of a degree. The results were compared for each group, paying special attention to the change in kyphotic angle or translation at maximum follow-up, the change during the period the halo was applied and the change over the period after the halo was removed. It was decided that the rates of change would be more representative of real progress due to the variation in exact follow-up dates.

\section{Patient questionnaire}

The literature was reviewed regarding complications of halo use. The most comprehensive of these was published by Garfin et al. ${ }^{5}$ Complications included pain, pin loosening, pin site infection, neurological injury, aphagia secondary to anchoring of a pin into the masseter, piercing the inner table of the skull, disfiguring scars at pin sites, and the development of pressure sores beneath the vest. ${ }^{5}$ A questionnaire was developed which assessed the presence or absence of particular symptoms as outlined in this study. Patients were also asked to rate the effect of the halo on their daily activities using a four-point scale, ranging from 'no effect at all' to 'severe limitation of activities' (Figure 1). Patients were also asked to assess the intensity of their pain and discomfort while wearing the halo using a four-point scale, ranging from 'no discomfort' to 'mild', 'moderate' or 'severe' pain (Figure 2). Finally, the patient was asked to rate his or her clinical outcome (Figure 3). Ratings described outcome as 'poor', 'good', or 'excellent' with respect to pain relief. A 'poor' rating indicated minimal improvement, while a rating of 'good' indicated definite, but not complete pain relief. An 'excellent' rating was reserved for cases in which the patient claimed to have complete resolution of pain. Patients were interviewed by telephone or

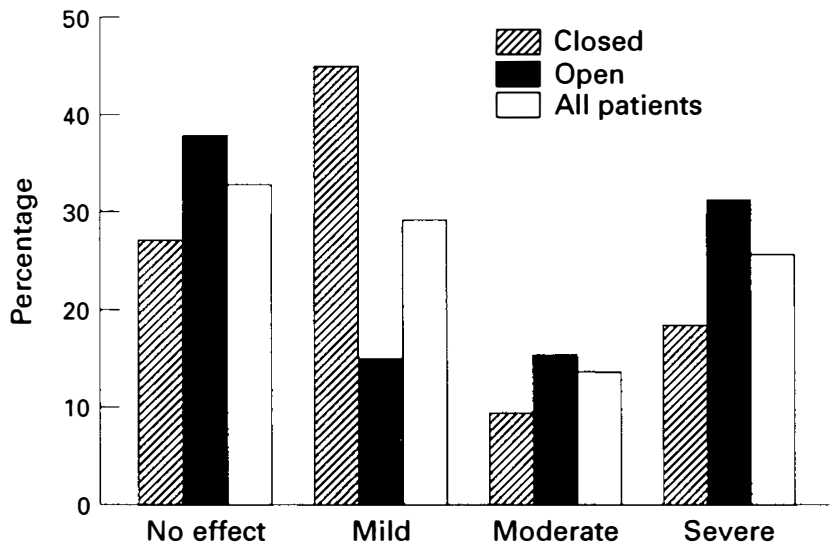

Figure 1 Effects of halo on daily activities

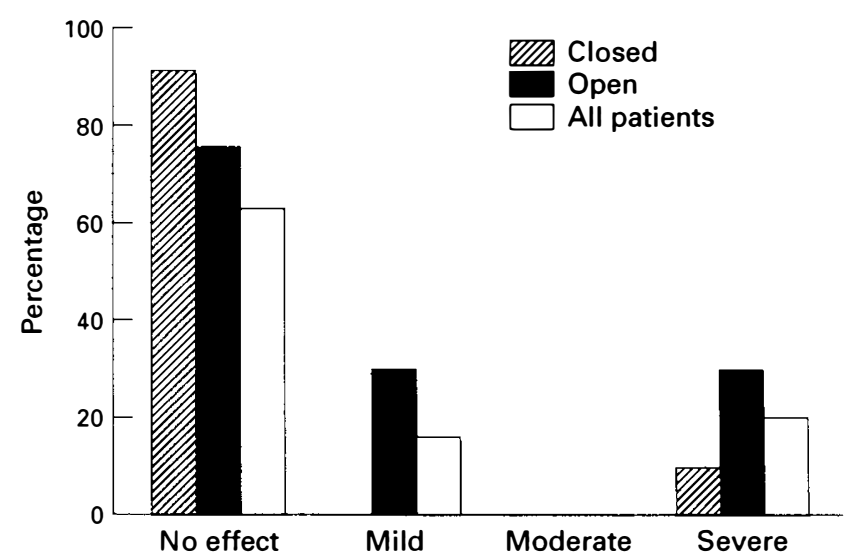

Figure 2 Severity of pain (in halo) as reported by patient

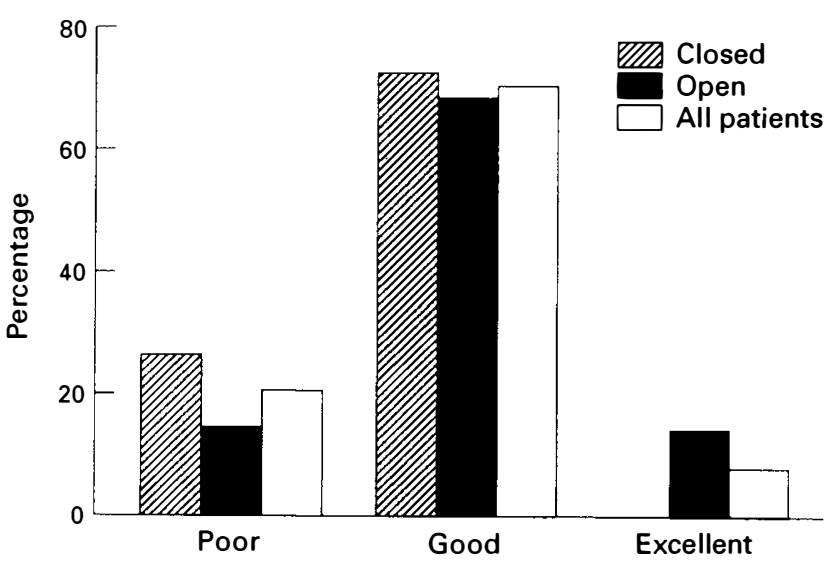

Figure 3 Outcome (pain relief) as reported by patient

in person, and their responses were recorded in a database. Data were analyzed by halo design (open versus closed), injury type, complications, treatment, and radiographic findings. 


\section{Results}

Eleven patients were treated with the closed ring, and 13 with the open ring. Ten females and 14 males were treated. There were five females and six males in the open ring group, and five females and eight males in the closed ring group. Mean age at halo application was 46 years (range 5 years, 8 months -78 years).

The type of injury varied widely between the groups. The majority of the closed ring group (six patients) presented with non-traumatic etiology, ie spondylosis, stenosis, or myelopathy. Odontoid fractures were the most common traumatic injury (two patients). Of the remainder, subluxation of the facet joint, fracture of a vertebral body, and burst fracture of a vertebra were each represented by individual cases. In the open ring group, two patients presented with degenerative disease. Other etiologies were fracture dislocation (1), odontoid fracture (5), and subluxation of a facet joint (3). There was a single case of pedicle fracture, and one case of fracture with subluxation of $\mathrm{C} 2$ onto $\mathrm{C} 3$. There were no cases of neoplasm. A mean of 1.8 spinal levels were involved (range 1-4).

Of the eight patients who presented with degenerative etiology, four demonstrated signs of frank myelopathy due to multiple level cervical stenosis. Four were neurologically intact. A total of seven patients suffered dens fractures. All were neurologically intact; as noted above the three displaced type II fractures were treated with Brooks arthrodesis, and the three minimally displaced type III fractures and one type II nondisplaced fracture were treated primarily with the halo. One patient with a unilateral facet fracture and subluxation, in the closed ring group, suffered a C6 root injury manifested by biceps and wrist extensor weakness; this resolved following reduction. The remaining two fracture patients in the closed group, one with a sagittal fracture of the body and one with a burst fracture at C6 were neurologically intact, and complete at the $\mathrm{C} 6$ level, respectively. The one patient in the open group who suffered a fracture dislocation at C4-5 was neurologically complete at that level. Three patients with facet fractures and subluxation in the open group, one treated operatively, and two primarily in a halo suffered transient root lesions, as manifested by dysesthesias and motor weakness at the level of the facet fractures. The patient who suffered a pedicle fracture, and was treated primarily with an open ring halo was neurologically intact. The patient in the open ring group suffering from $\mathrm{C} 2-3$ subluxation was taken to the operating room for open reduction and stabilization; neurological status was that of a complete spinal cord injury.

The halo was used as the primary form of treatment in 10 patients (four closed, six open). In the remainder, the halo was used as postoperative support. One patient each underwent anterior fusion with the iliac crest autograft, anterior fusion with a fibular strut allograft, and anterior fusion with plating and allograft. There was a single case in the closed ring group involving anterior and posterior fusion using both a fibular strut allograft and iliac crest allograft. Other procedures included posterior fusion with iliac crest autograft (two patients), corpectomy and fusion with fibular strut allograft (three patients), corpectomy and fusion with iliac crest autograft (two patients), and Brooks posterior fusion (three patients). Additional surgical intervention was required in four of the cases. These cases were evenly distributed between closed and open groups.

Maximum follow-up ranged from 3-20 months, with a mean of 7.5 months. The number of days which the halo remained in place ranged from 48 to 111 , with a mean of 81.9. There were no significant differences between the closed and open ring groups regarding follow-up periods or length of halo application.

\section{Radiographic data}

There were no differences between closed and open ring groups for changes in kyphotic angle or translation over specified intervals (time to maximum follow-up, duration of halo placement, and the time from halo removal to maximum follow-up). Similarly, when values for the kyphotic angle and translational change were divided by the time interval between follow-up periods to determine a more standard rate of change, data revealed no significant difference between groups. Independent associations were made between absolute change in translation after halo removal and tobacco use $(P=0.041)$. Rate of change, however, was not associated with tobacco use $(P=0.123)$.

Analysis was similarly conducted comparing surgical approach. A single case-a four level anteriorposterior fusion with fibular strut allograft and iliac crest autograft, treated in an open halo-was significantly different from the rest of the group in terms of kyphotic change $(P=0.0012)$ and translation $(P=0.0053)$ while in the halo. There was no significant change from other cases at maximum follow-up, however. There were no significant differences between patients who had undergone repeat surgery and patients for whom the initial therapy was adequate when radiographic data was compared between those groups. No correlations were found between the number of levels of involvement and the radiographic findings.

\section{Complications (Figure 4)}

Complication rates for this study were higher than expected, based on the literature. Pin site infection as proven by culture and sensitivity or surrounding cellulitis occurred in $31.3 \%$ of all sites and pin loosening as defined by torque wrench reading of less than 6 inch/pound occurred in $75 \%$. Pin site infection and pain were more common in the open group, while scarring and loosening occurred more frequently in the closed group. Complaints of pain were significantly more frequent among patients treated with the open ring $(P=0.032)$, with $62 \%$ of these patients complaining of noticeable pain during the time they wore the halo. Conversely, $91 \%$ of closed ring patients claimed they were pain-free in that interval. When asked to rate 


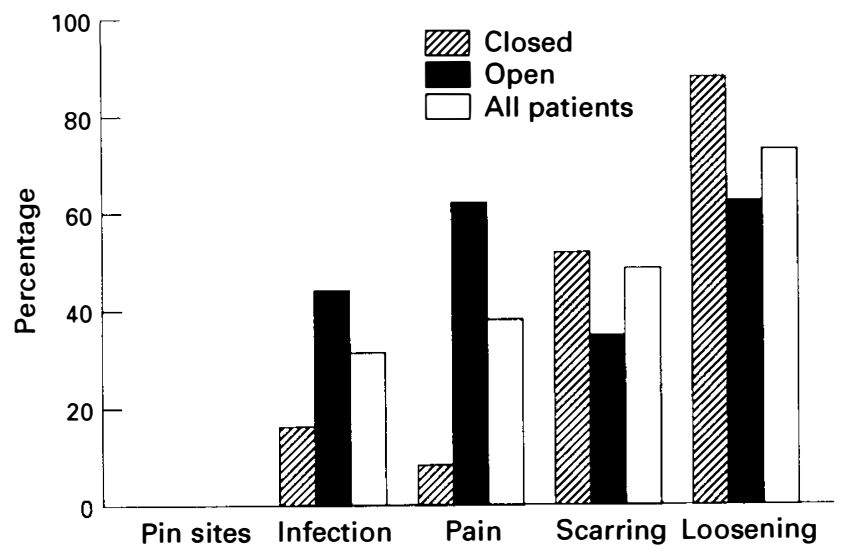

Figure 4 Rates of common complications

the intensity of their pain, however, patients in the open ring group were evenly divided between ratings of no pain (five patients), mild pain (four patients), and severe pain (four patients). Closed ring patients were heavily weighted toward the low end of the pain scale, with all but one of the patients claiming to be pain-free. This patient registered a complaint of severe pain (Figure 2).

Most patients felt that their clinical outcome was favorable with respect to pain relief (Figure 3). Overall, five patients were dissatisfied with their outcome. There was no statistical difference between open or closed groups in their responses. There were also no significant differences between the two groups when asked to evaluate the impact of the halo on their daily activities (leaving the home, going to public places, riding in a car). Fewer than half of the patients overall $(38 \%)$ felt that the halo was moderately or severely restrictive (Figure 1). While there was no strict correlation with restrictiveness and pain score, the patients in the open group, which had higher pain scores overall, tended to be more restricted (moderately of severely) than patients in the closed group. When examined by injury type, treatment modality, or the need for repeat surgery there was no significant difference between groups. There were no significant correlations between reports of outcome or pain intensity in relation to radiographic data. There were also no significant correlations between pin loosening and radiographic outcome. Presumably, this complication would allow motion within the halo and thus permit destabilization at the site of injury. Finally, there was no significant correlation between pin loosening and pain severity.

\section{Discussion}

These data indicate that the closed and open ring halo devices are very similar in terms of stability, as measured by radiographic criteria. With the ease of application of the open ring halo, the advantages of the open ring seem particular attractive. According to these data, stability is afforded by either design, regardless of the levels involved or the etiology of injury.

The majority of patients in the closed ring group, six, presented with a non-traumatic etiology. Only two patients, however, in the open ring group suffered from degenerative disease. Given a higher predominance of trauma in the open ring group, it is likely that these patients were more inherently unstable. However the halo was used as a primary form of immobilization in four patients in the closed group and six in the open; all of these were trauma patients. Thus, while the absolute number of trauma patients was greater in the open group, in fact, only two more trauma patients (six versus four) were treated primarily with an open rather than a closed halo. The remainder of trauma patients in the open group were, in fact, postoperative. While this certainly, by no means, eliminates a possible bias regarding patient selection in open and closed groups, it does appear that the groups are roughly comparable for the purpose of preliminary analysis of halo stability. In any event, if a more 'unstable' group was being treated by the open ring, then the fact that no significant quantitative changes in radiographic findings were found in this preliminary study would suggest that the newer design sacrifices little in terms of rigidity.

On examination of the radiographic data, the standard deviations were found to be quite broad with respect to the mean. Most values, however, were well within expectations. Since a computer enhanced digitizer was used to determine values for kyphotic angle and translation, precision was not a limiting factor. The best agreement one could typically expect with unassisted drafting is 4 degrees for kyphotic angle, and 3-4 $\mathrm{mm}$ for sagittal translation. ${ }^{6}$ With digitization, the calculated results for translation were so minute that an artificial standard had to be developed. For instance, rate of translational change (per day) had to be reported in micrometers to achieve whole number values. If the time intervals were extended to months rather than days, the means would still be expressed in tenths of a millimeter, or at worst, in units of a single millimeter. The radiographic measurements were thus very reliable. Interestingly, in only one instance was radiographic change significant, with a change in absolute sagittal translation post-halo removal being greater in smokers than non-smokers. This is in accordance with similar reports of other deleterious effect of cigarette smoking (eg a higher pseudarthrosis rate $)^{7}$ reported in the literature and is presumably related to vascular or biochemical interference with bony healing processes.

Complication rates for this study were far greater than those reported by Garfin et al $(n=59)$ : a pin site infection rate of $20 \%$, pin loosening $36 \%$, and scarring at pin sites $9 \% .{ }^{5}$ Particularly with regard to pin loosening and scarring, incidences for both closed and open groups were double or triple the expected rates. Glaser et al reported 'pin track problems' in 14 of 245 cases $(6 \%)$; these are identified as drainage, with no mention of loosening criteria or total number of pins implanted. ${ }^{8}$ Tator et al reported pin site infection in 
four of 59 patients $(7 \%)$ and loosening in five of 59 $(8 \%)$; again no mention is made of specific criteria for these determinations. ${ }^{9}$ In their report of 188 patients, Chan et al considered a pin to be loose only when re-application was required (four patients); infection in 11 patients was reported only when pin change was needed. ${ }^{10}$ In the present study, the rate of pin site infection was $31.3 \%$, of pin site pain $43.8 \%$, scarring $42.7 \%$, and loosening $75 \%$. However, compared to the report of Garfin et al,${ }^{5}$ more patients in this study were pain-free in the halo $(62.5 \%$ versus $38 \%)$. Given these findings, it may in fact be the case that criteria for loosening were more strictly applied to the present study. Also, the pin care regimen may have contributed to the loosening. As others have noted, ${ }^{5}$ cleansing more frequently than every other day may lead to low grade infection due to manipulation of the wound site. This certainly could have been the case in the present study. It had been our impression that more frequent cleaning was desirable; this certainly does not appear to be the case. Despite the high complication rates, the overall outcome for both groups was good.

The patients in the closed group tended to report less pain than the patients in the open group. The reasons for this trend are not clear; it could be due to the relatively decreased rigidity of the open ring. An open device is subject to stresses which tend to further spread the open end and concentrates stresses on the apex of the ring. In the Bremer design, this 'wishbone' effect could occur in two planes, with the effect being spreading of the device posteriorly and placing traction on the pins (Figure 5).

There are a number of limitation to this study. This is a retrospective study with a small number of patients. However, all patients did have accurate, recent accounts of their progress with the halo in places. The relatively brief 8 -month mean follow-up period could, in fact, be extended. Ideally, the same patients can be re-interviewed in 1 or 2 years, not only to verify the initial data, but to generate more comprehensive data on clinical outcome. Arguably, however, patient report of the 'in halo' experience are more accurate with shorter follow-up. Thus, this aspect of the current data would seem to be sufficient, with the large qualification that all these data are subjective in nature. Indeed, the significant difference in the clinical category between open and closed groups was pain severity-an extremely subjective issue. Regarding radiographic data, the use of computer digitization and average rate of change produced data of a very high degree of accuracy; this technique could be applied to future films to update radiographic data with relative ease and verify objective outcome long-term.

Based on these data, it is difficult to determine which device is superior. While the open ring is easier to apply and thus minimized the possibility of injury to the unstable cervical spine, the higher rate of 'in halo' pain is of concern. If, in fact, this is related to the less rigid design, than a higher rate of pin loosening would be expected. The loosening rate, however, was greater in the more rigid closed ring group. The open ring group
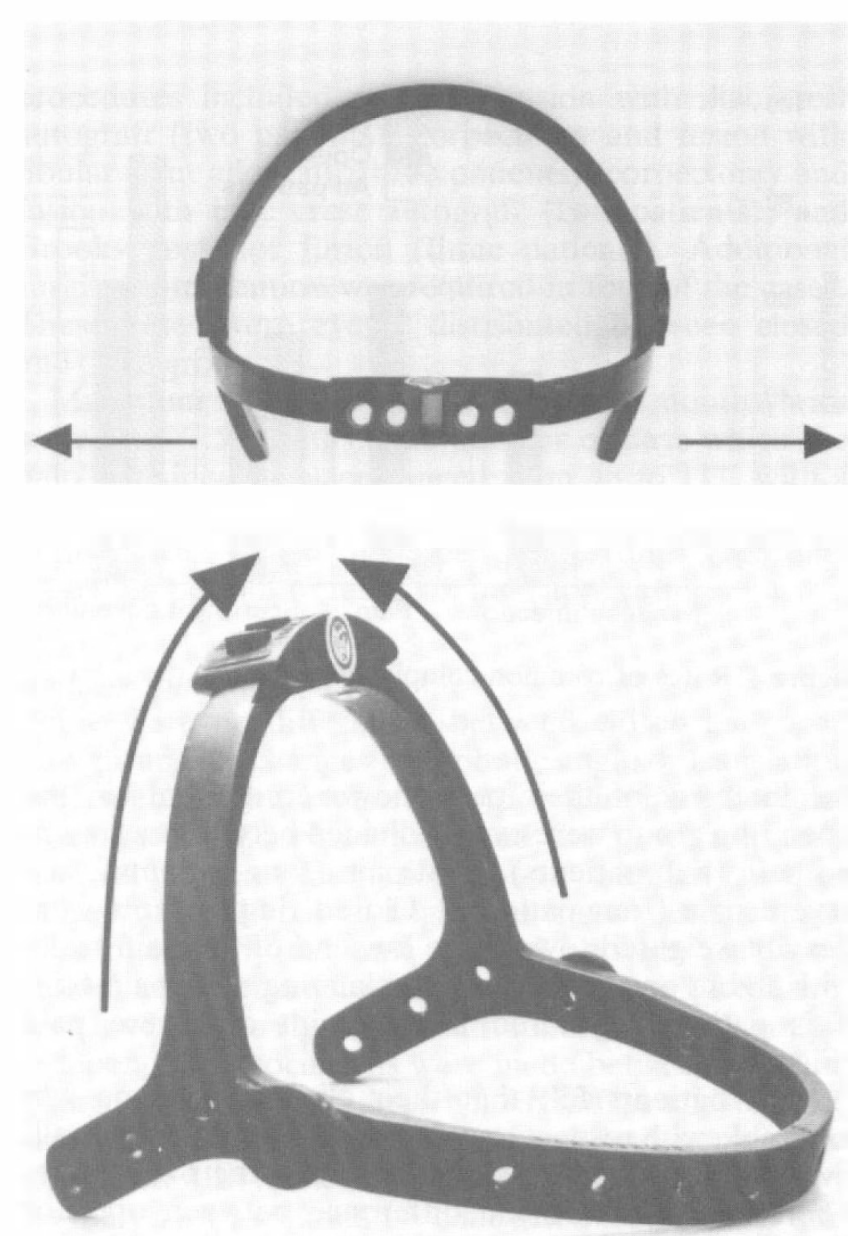

Figure 5 The 'wishbone' effect. An open ring structure can, theoretically, flex and spread, thus opening like a wishbone. Clearly, biomechanical testing is required to determine whether this hypothetical mechanism occurs with clinically relevant loads

did have a higher rate of infection; the reasons for this are unclear, but could be related to telescoping of the pin. Additionally, the higher rate of pin tract infection overall, could be explained by over zealous pin tract care. If the relatively increased flexibility of the open ring accounts for increased pain, then little practical advantage of the open ring system is apparent unless an 'optimum rigidity' can be defined. With newer, more rigid open ring designs becoming available for use, further assessment is clearly required. The development of a system whereby the ring could be 'closed' following application is, likewise, of potential interest if in fact, the most rigid system is clinically, the most desirable.

\section{References}

1 Perry J, Nickel VL. Total cervical spine fusion for neck paralysis. J Bone Joint Surg Am 1959; 41: 37-39.

2 Koch RA and Nickel VL. The halo vest. An evaluation of motion and forces across the neck. Spine 3: 103-107.

3 Anderson PA et al. Failure of Halo vest to Prevent in vivo motion of patients with injured cervical spines. Spine 1991; 16: 
5501-5505

4 Johnson RM, Owen JR, Hart DL, Callahan RA. Cervical orthoses, a guide to their selection and use. Clin Orthop 1981; 154: 34 .

5 Garfin SR, Botte MJ, Nickel VL. Complication of the use of the halo fixation device. J Bone Joint Surg Am 68: 320-326.

6 Dawson EG, Smith RK, McNeice GM. Radiographic evaluation of scoliosis: a reassessment and introduction of the scoliosis chariot. Clin Orthop 1978; 131: 151-155.

7 Brown CW, Orme TJ, Richardson HD. The rate of pseudoarthrosis (surgical nonunion) in patients who are smokers and who are non-smokers - a comparison study. Spine 1986; 11: $942-943$

8 Glaser JA, Whitehall R, Stamp WG, Jane JA. Complications associated with the halo-vest. J Neurosurg 1986; 65: 762-769.

9 Tator $\mathrm{CH}$ et al. Halo devices for the treatment of adult cervical spinal cord injury. In: Tator $\mathrm{CH}$ (ed). Early Management of Acute Spinal Cord Injury. Raven Press: New York 1982, pp $231-256$

10 Chan RC, Schweigel JF, Thompson GB. Halo-thoracic brace immobilization of 188 patients with acute cervical spine injuries. J Neurosurg 1983; 58: 508-515. 